\title{
Research of the key technology of AIS base station web system
}

\author{
Boran Yu, Shufang Zhang
}

\author{
Navigation Institute, Dalian maritime university, Dalian, China \\ Navigation Institute, Dalian maritime university, Dalian, China \\ David_cs@126.com,sfzhang@dlmu.edu.cn
}

\begin{abstract}
Keywords: AIS; message protocol; base station; independent design
\end{abstract}
\begin{abstract}
This essay is to research the key technology of AIS base station web system. After specifically analyzing the AIS system and AIS message protocol, it majorly discusses the independent design of the AIS base station web system, which consists of node servers, one control center, and remote accesses; the system has been tested by the Aton department in Dalian. The result of test shows that this system does well in operating and administrating the AIS base station, and gets high evaluation of the Aton department.
\end{abstract}

\section{Introduction}

With the development of the AIS (automatic identification system) and wider usage of AIS application, there appear more and more AIS devices and base stations leading to a rapidly increasing amount of information [1]. Thus the usage of AIS information has been a lot widened than before, and the work that staffs have to do about it has been significantly increased. As a result, as a very important part of the AIS system, the development of AIS base station web system is becoming increasingly significant [2]. So far, AIS tech has become the key part of the marine communication, the application of which has become widely used throughout the world. However in our country, the development of the AIS base station web still remains relatively fallen behind, a lot of devices depend on importing [3], for instance, AIS base station web software which has been widely used in China is made by SAAB company, which shows an comparison between the domestically increasingly need of the AIS base station web system and the relatively deficiency of the relevant technology. Mentioned above, researching on the AIS base station web system has become a vital scientific task. The subject of this essay is raised on this circumstance.

\section{AIS system analysis and AIS message analysis}

AIS system analysis. AIS is an automatic consecutive broadcasting system by time division multiple access which works on the maritime VHF frequency [4]; It can implement exchanging and alternating of the on shore and off shore information like the vessel identification, position, course and speed of the vessel; Aligning with GPS broadcasting, it can transmit information including ship dynamic information and ship static information through VHF to the ships and base stations around [5]. So the neighboring ships and base stations are able to get this information, and provide assist information of collision prevention to reduce the accident rate [6].

AIS message analysis. The transmitting and interpreting of IEC 61162-1 supported packaged binary data usually needs the data and information outside the IEC 61162-1 protocol. IEC61162-1 protocol contains the coding, decoding and constructing information [7]. For AIS, the meaning of the binary data can be referred to from ITU-R M.1371 standard [8].

AIS international standard involves some forms, which specify the construction of the AIS message position by position. The following figure 1 shows the different parts of the "radio pack" created and broadcasted by AIS. Information bit is added with some additional bit for the radio signal broadcasting. These additional bit can be erased in the AIS receive unit. Only the data bit remains in the character string involved in the VDM sentence. The erased bit involves foreword, 
is shown in the following Fig. 1.

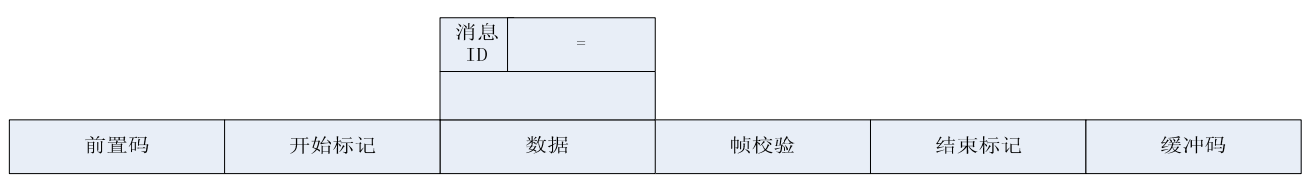

Fig. 1 the bit structure of AIS message

\section{AIS base station web system general design}

This software system consists of several node servers, one control center and several remote accesses. Considering about the requested data secrecy, this system is using the AIS local network. Node servers get the AIS data from different AIS base stations through serial port then transmit the data to the collecting server involved in the center control system through AIS network. After the data is decoded, backup server does the data backup while web server restores the data in the database. And then the data release server releases the data on the website for users to monitor and use through remote accesses like desktops and laptops. The framework of the system general design is shown in the following Fig. 2.

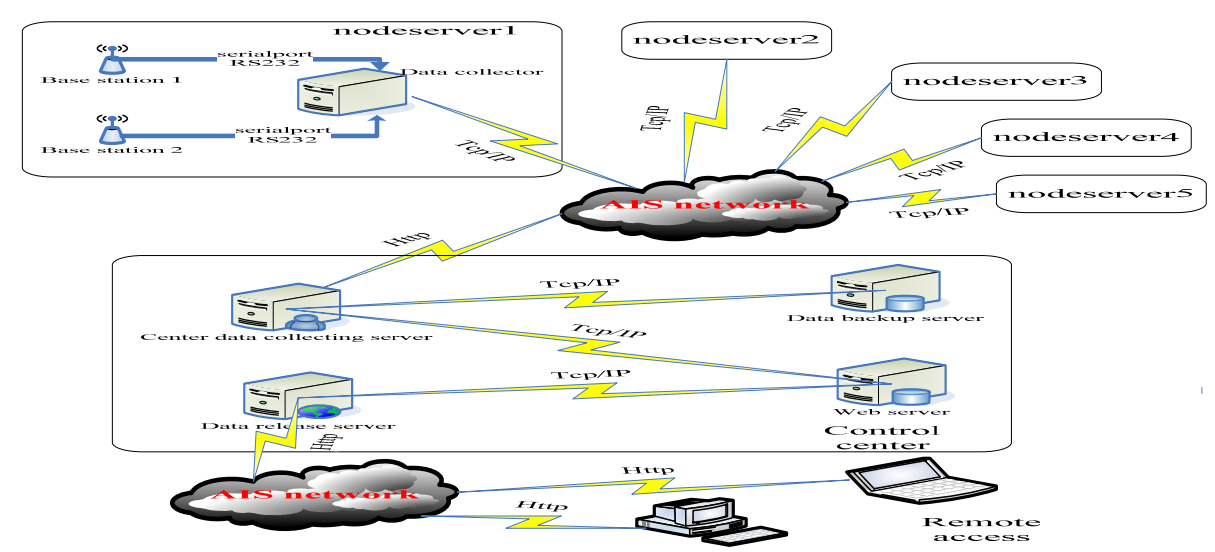

Fig. 2 framework of the AIS web system

\section{Subsystem design}

AIS base station web system implements the integrate process with 3 subsystems, which are node server subsystem, control center subsystem, and remote access subsystem. They're working as integrity combined by the AIS local network.

Node server system. The node system is established at the base station equipment rooms, the job of which is to receive the AIS data through serial port from the AIS base station with baud rate of 38400. They are all connected to the control center by the AIS network. Expect for collecting and uploading data, the node systems can also do data backup for the latest 7 days in case of the unexpected situations like power fails etc.

Control center system. Control center system is always laid in the AIS device center of the local Aton department. It is the centerpiece of the whole system which contains 4 different servers that are data collecting server, data backup server, web server and data release server. Data collecting server has two jobs. First, it needs to receive the data sent by the node server system. And then it needs to decode the AIS message to get the information like latitude and longitude of the devices like vassals, Atons and base stations. Then the data backup server gets the information to do data backup and the web server gets the data to save it in the database which is installed at the server. The data release server then gets the new information and releases it on the website for users to use. The website uses the MVC structure that allows the users to add, delete, modify and search the relative information.

Remote access. The staffs can log on the website by entering user name and password through remote accesses like desktops and laptops in order to observe the AIS data and remote control the AIS base station. The system allows 2 groups to log on it with different authorities as operator and 
administrator. The operators can observe and operate the data, check the link connection of the AIS base stations, while the administrators can add or delete the base stations to be controlled, set the authorities of different staffs as well as set the alarm levels of different kind of breakdown of base stations or data link problems.

\section{AIS base station web system test}

AIS base station web system is important in the whole AIS system, the stability and robustness of which is directly relevant to the safety of ship sailing and efficiency of the authority administrating. The test of this new developed AIS base station web system is done at the relevant department that authorizes the Aton devices for several months. And it's been debugged and improved several times during the test. From the feedback of this department as well as data inspection and analysis, it has proved that the system has performed perfectly in both data accuracy and system stability. It has won high affirmation of the relevant department.

Part of the test result of the AIS base station web system is as shown in the following Fig. 3.

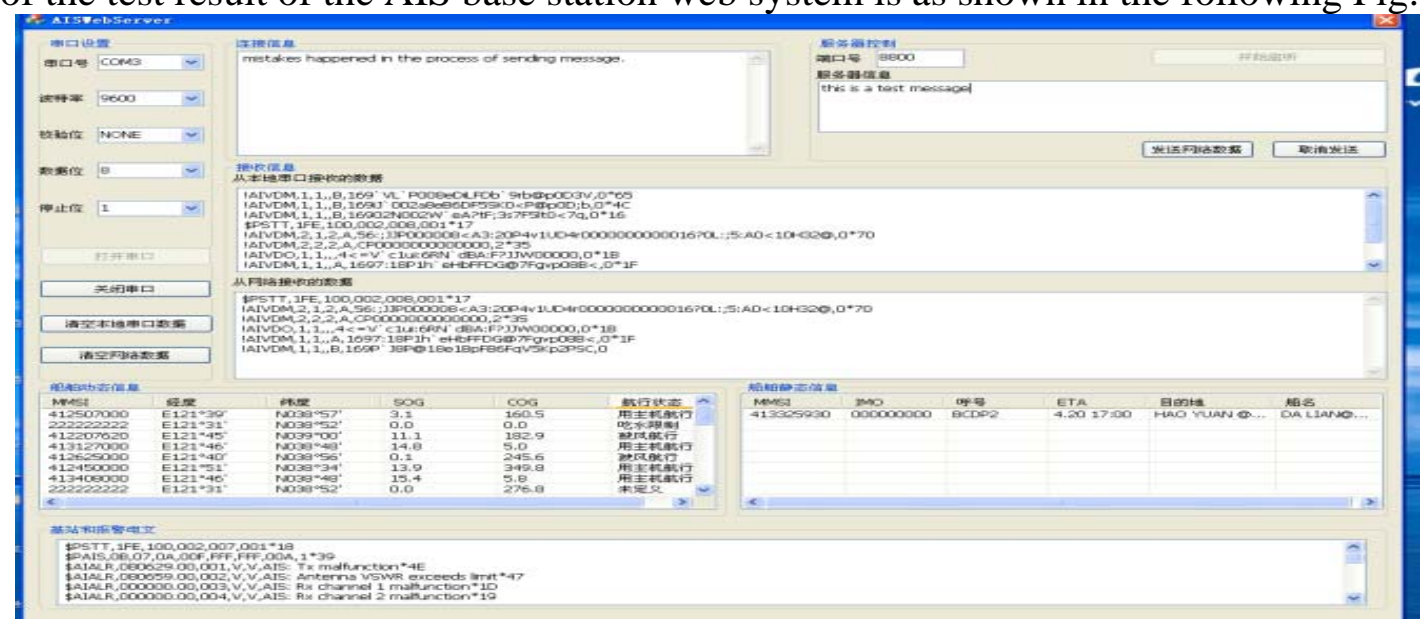

Fig. 3 part of the test result of the AIS web system

\section{Summary}

AIS system is significant in the domain of ship management, and the development of the AIS base station is a very important part in the whole system, the stability and robustness of which is vital to the application of the AIS system. This paper has researched the AIS base station web system based on the analyzing of the AIS web and the on shore, off shore departments' requirement; specifically designs a integrate AIS base station web system. Through the practical test by using the SAAB base station in the navigation lab of the DMU, it has proved that this system is able to implement the function of AIS web system with high stability, robustness and high efficiency.

\section{References}

[1] Qing Hu, and Shufang Zhang, "Public wireless mobile IP network-based AIS technique in E-Navigation”, DMU journal, 2008, pp. 46-50

[2] Chang, S.J., "Development and analysis of AIS applications as an efficient tool for vessel traffic service”, in 2004 IEEE, pp. 2249-2253

[3] JiaChun Zheng, VTS information broadcasting techbology, Navigation technology, 2004, pp. 32-34

[4] Ershen Wang, and Shufang Zhang, "Design of information display and control instrument based on SoC for AIS and GPRS monitoring system” in 2008 3rd IEEE Conference on Industrial Electronics and Applications, ICIEA 2008, 2008, pp. 32-35 
[5] ZunShan Zhou, and Lei Li, “Usage of AIS in Maritime management”, Navigation Technology, 2005, pp. 33-34

[6] Tiekun Tu, “The noticeable events in the practical usage of AIS”, journal of jimei university, 2005, pp. 37-41

[7] Richard Kjellberg. Analysis of an AIS Implementation in Tokyo Bay, 2001.

Ivor Horton. Beginning visual c++. Wiley Publishing, 2010. 